\title{
CSF Flow in the Brain in the Context of Normal Pressure Hydrocephalus
}

W.G. Bradley Jr

\begin{abstract}
SUMMARY: CSF normally flows back and forth through the aqueduct during the cardiac cycle. During systole, the brain and intracranial vasculature expand and compress the lateral and third ventricles, forcing CSF craniocaudad. During diastole, they contract and flow through the aqueduct reverses. Hyperdynamic CSF flow through the aqueduct is seen when there is ventricular enlargement without cerebral atrophy. Therefore, patients presenting with clinical normal pressure hydrocephalus who have hyperdynamic CSF flow have been found to respond better to ventriculoperitoneal shunting than those with normal or decreased CSF flow. Patients with normal pressure hydrocephalus have also been found to have larger intracranial volumes than sex-matched controls, suggesting that they may have had benign external hydrocephalus as infants. While their arachnoidal granulations clearly have decreased CSF resorptive capacity, it now appears that this is fixed and that the arachnoidal granulations are not merely immature. Such patients appear to develop a parallel pathway for CSF to exit the ventricles through the extracellular space of the brain and the venous side of the glymphatic system. This pathway remains functional until late adulthood when the patient develops deep white matter ischemia, which is characterized histologically by myelin pallor (ie, loss of lipid). The attraction between the bare myelin protein and the CSF increases resistance to the extracellular outflow of CSF, causing it to back up, resulting in hydrocephalus. Thus idiopathic normal pressure hydrocephalus appears to be a "2 hit" disease: benign external hydrocephalus in infancy followed by deep white matter ischemia in late adulthood.
\end{abstract}

ABBREVIATIONS: ACSV = aqueductal CSF stroke volume; DESH = disproportionately enlarged subarachnoid space hydrocephalus; DWMI = deep white matter ischemia; ISP = interstitial space; NPH = normal pressure hydrocephalus; PC = phase-contrast; SAS = subarachnoid space

$\mathrm{T}$ he classic teaching is that cerebrospinal fluid is formed primarily in the choroid plexus within the ventricles at a rate of $500 \mathrm{~mL} /$ day. It flows primarily out of the ventricular system via the foramina of Lushka and Magendie into the subarachnoid space (SAS). Once in the SAS, the CSF flows either down around the spinal cord or up over the cerebral convexities, eventually being primarily absorbed by the arachnoid granulations (macroscopic) and arachnoidal villi (microscopic) on either side of the superior sagittal sinus. This CSF resorption pattern was based on tracer studies performed many years ago by using large molecules.

Recently, a microscopic flow of CSF has been described that functions like the lymphatic system in other parts of the body. ${ }^{1}$ It has been shown, by using gadolinium and small fluorescent tracers in mice,

From the Department of Radiology, University of California, San Diego, San Diego, California.

Please address correspondence to William G. Bradley Jr, MD, Department of Radiology, University of California, San Diego, 200 Arbor Dr, MC 8224, San Diego, CA 92103; e-mail: wgbradley@ucsd.edu

- Indicates open access to non-subscribers at www.ajnr.org

http://dx.doi.org/10.3174/ajnr.A4124 that CSF enters the perivascular Virchow-Robin spaces surrounding the arteries from the SAS. ${ }^{2}$ Lining these arteries and arterioles are continuous astrocytic endfeet - the glia limitans — which have high concentrations of aquaporin- 4 water channels, which transport CSF from the Virchow-Robin spaces into the interstitial space (ISP) of the brain. This microscopic CSF flux picks up waste products, (eg, solutes and $\beta$ amyloid) and exits the ISP via the aquaporin- 4 channels lining the Virchow-Robin spaces surrounding the exiting veins. Given the lymphatic-like function and involvement of the astroglia, this fluid flow has been dubbed the "glymphatic system."

The movement of CSF into the brain via the Virchow-Robin spaces is powered by arterial pulsations. ${ }^{3}$ Given that these are reduced in some elderly patients, the glymphatic flux is decreased; this decrease may lead to the accumulation of $\beta$ amyloid and potentially contribute to Alzheimer disease. Water moves freely into the ISP from the SAS but not from the ventricular compartment into the ISP, unless there is increased intraventricular pressure. From there, the CSF can drain via the aquaporin- 4 channels into any venous structure, (eg, the vein of Galen, straight sinus, or superficial cortical veins; ie, not necessarily the arachnoidal villi).

AJNR Am J Neuroradiol 36:831-38 May 2015 www.ajnr.org 

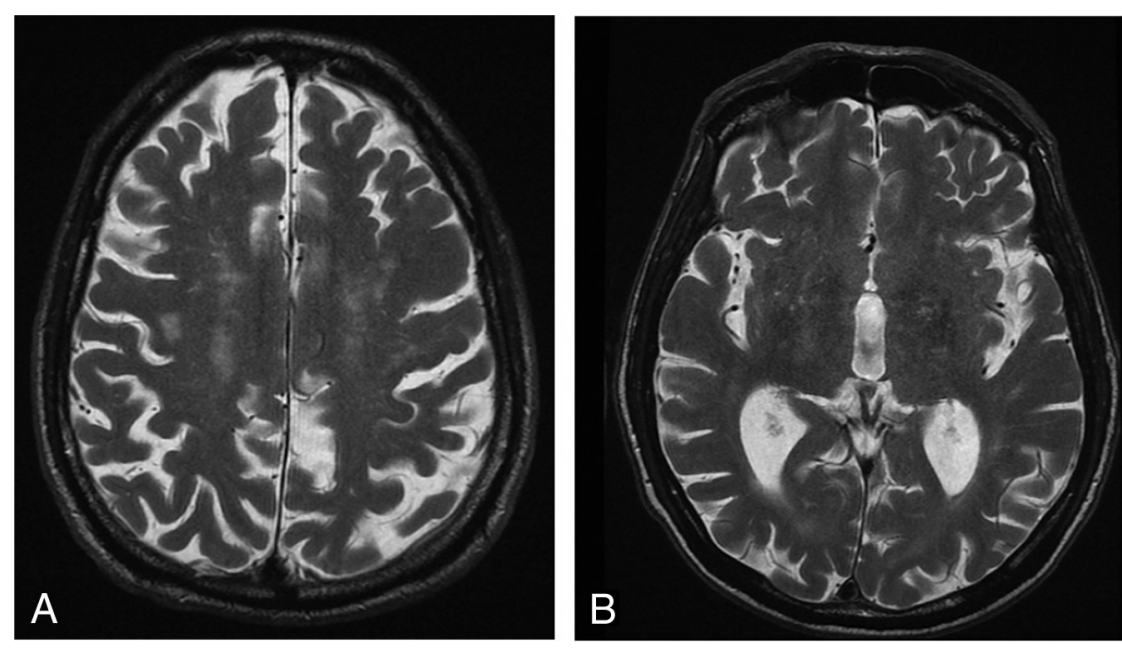

cranial nerves in the basal cisterns. Regardless of the exact percentage of CSF produced by the brain versus the choroid plexus, the bulk flow of CSF is out of the lateral ventricles via the foramen of Monro through the third ventricle and aqueduct and then through the fourth ventricle.

Obstruction of the outflow of CSF proximal to the outlet foramina of the fourth ventricle was termed "obstructive hydrocephalus," while obstruction distal to the foramina of Lushka and Magendie was termed "communicating hydrocephalus" by Dandy $>100$ years ago. Most obstructive hydrocephalus in adults is due to
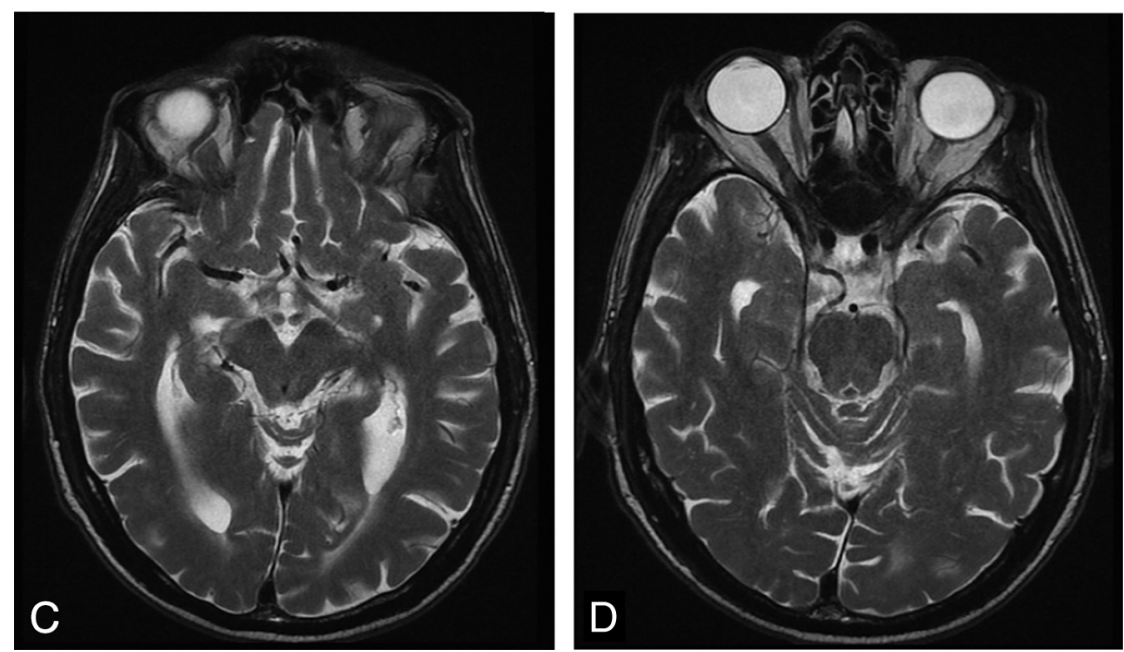
tumors obstructing the outflow of CSF upstream of the outlet foramina of the fourth ventricle. Most cases of communicating hydrocephalus are due to subarachnoid hemorrhage or meningitis, the former obstructing the arachnoidal villi and the latter often obstructing more proximally at the level of basal cisterns, particularly with viscous fungal, tubercular, or other granulomatous meningitides. A subset of communicating hydrocephalus seen in the elderly is termed "normal pressure hydrocephalus" (NPH) (Fig 1) and is defined by the clinical triad of gait disturbance, dementia, and incontinence.

A subset of communicating hydro-

FIG 1. NPH on T2-weighted fast spin-echo, axial images. A, A section through the centrum semiovale demonstrates deep white matter ischemia. $B$, A section through third ventricle shows loss of the waist due to slight enlargement with minimal CSF flow void. $C$ and $D$, Sections through the aqueduct and upper fourth ventricle show CSF flow void. Although less conspicuous than in the past by using conventional spin-echo, the CSF flow void sign is now more specific for hyperdynamic flow, albeit less sensitive.

\section{Normal Macroscopic CSF Flow}

Superimposed on the slow egress of CSF from the ventricles to the SAS is a more prominent pulsatile motion due to the beating of the heart. During systole, blood flows into the brain causing it to expand inward, compressing the ventricles, and outward, compressing the cortical veins and SAS. The inward expansion leads to pulsatile outflow of CSF through the aqueduct and the rest of the ventricular system. This outflow results in a normal MR imaging CSF flow void in the aqueduct. ${ }^{4}$ The systolic expansion forces CSF and venous blood out of the fixed volume of the skull by the Monro-Kellie hypothesis. ${ }^{5}$ This process results in the systolic outflow of CSF at the foramen magnum and from there down the SAS of the spinal canal. During diastole, the volume of the brain decreases and CSF flows in a reverse direction through the aqueduct and the foramen magnum.

While most CSF is produced by the choroid plexus, recent evidence suggests that a portion of the CSF is made by the capillaries in the brain parenchyma. Similarly, it has been estimated that up to $20 \%$ of the CSF uptake occurs in the brain parenchyma or via the lymphatics near the cribriform plate or covering the posedly due to decret by "immature" arachnoidal granulations (Fig 2). Because their sutures are still open, such children present with a percentile head circumference growing at a faster rate than the percentile of body weight or body length and are referred for imaging to exclude a brain tumor. CSF accumulates over the frontal convexities as the head enlarges; this accumulation leads to a characteristic imaging appearance (Fig 2). Because this condition has been considered the result of immature arachnoidal granulations, which will eventually catch up to the production of CSF, these children do not need to be shunted - though recent findings suggest that this condition may not be as benign as previously thought (see below).

MR imaging is generally considered the best technique to evaluate hydrocephalus, partly because of its ability to image directly in the midsagittal plane and partly due to the various pulse sequences available. The best MR imaging technique to evaluate hydrocephalus is FLAIR, which is sensitive to the presence of interstitial edema (Fig 3). Immediately following obstruction, CSF production continues unabated and the amount of interstitial edema surrounding the lateral ventricles is at its greatest. While 


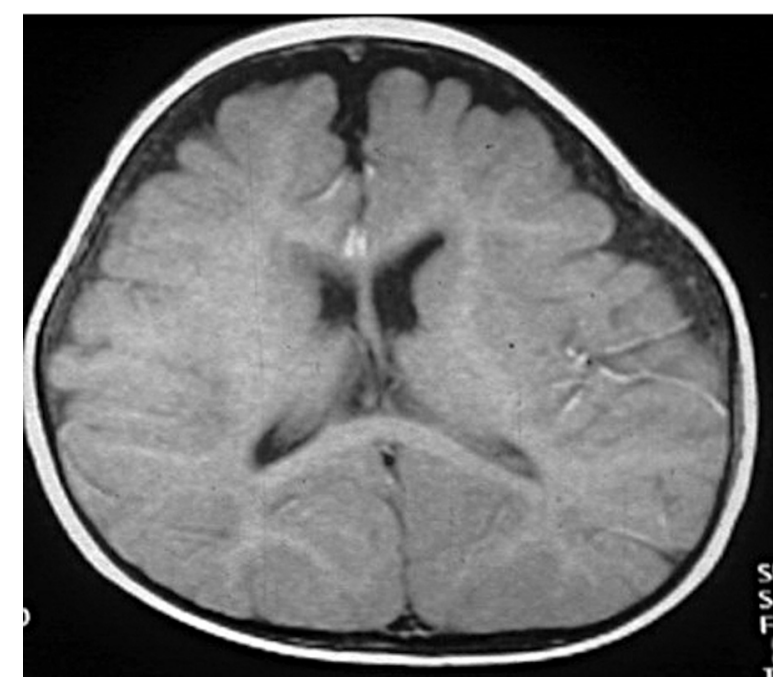

FIG 2. Benign external hydrocephalus in a 7-month-old infant with mild ventriculomegaly and increased CSF in the frontal subarachnoid space.

this feature is usually thought to be due to the pressure gradient across the ventricular wall forcing the CSF out transependymally, interstitial edema may also be due to CSF produced by the brain, which cannot get into the higher pressure ventricles. With time, the ventricles will enlarge to accommodate the increased intraventricular pressure and the pressure gradient will decrease, decreasing the amount of interstitial edema. Eventually the lateral ventricles will enlarge to the point at which the mean intraventricular pressure is normal and the interstitial edema resolves. At this point, the hydrocephalus is said to be "compensated" or "arrested." While the mean intraventricular pressure may be normal, the pulse pressure is often 6-8 times normal and is referred to as a "waterhammer pulse." The pounding of the lateral ventricles on the paracentral fibers of the corticospinal tracts, which supply the legs, may contribute to the gait disturbance in NPH.

\section{NPH}

Normal pressure hydrocephalus was first described by Hakim and $\mathrm{Adams}^{8}$ and Adams et $\mathrm{al}^{9}$ in 1965. At that time, the cause was not known-that is, the disease was considered "idiopathic." Since that time, patients with known causes of chronic communicating hydrocephalus have also been included as NPH. These known patients tend to be younger and respond better to shunting than those with the idiopathic variety, possibly because of poor historical selection criteria for the idiopathic form.

To be diagnosed with NPH, patients must first have enlarged ventricles and at least a portion of the clinical triad. The gait disturbance is usually the first symptom to appear, followed by dementia and last by urinary incontinence. The best response to shunting occurs earlier in the disease when gait is the primary symptom. In the first decade following the description of NPH in the literature, some patients who had only dementia were shunted, with obvious poor results, which led some to question the very existence of NPH. Today, up to $10 \%{ }^{10}$ of patients with dementia may have NPH and therefore may be treatable by shunting. Of course such patients will also have a gait disturbance.

When an elderly patient presents with a gait disturbance sug-
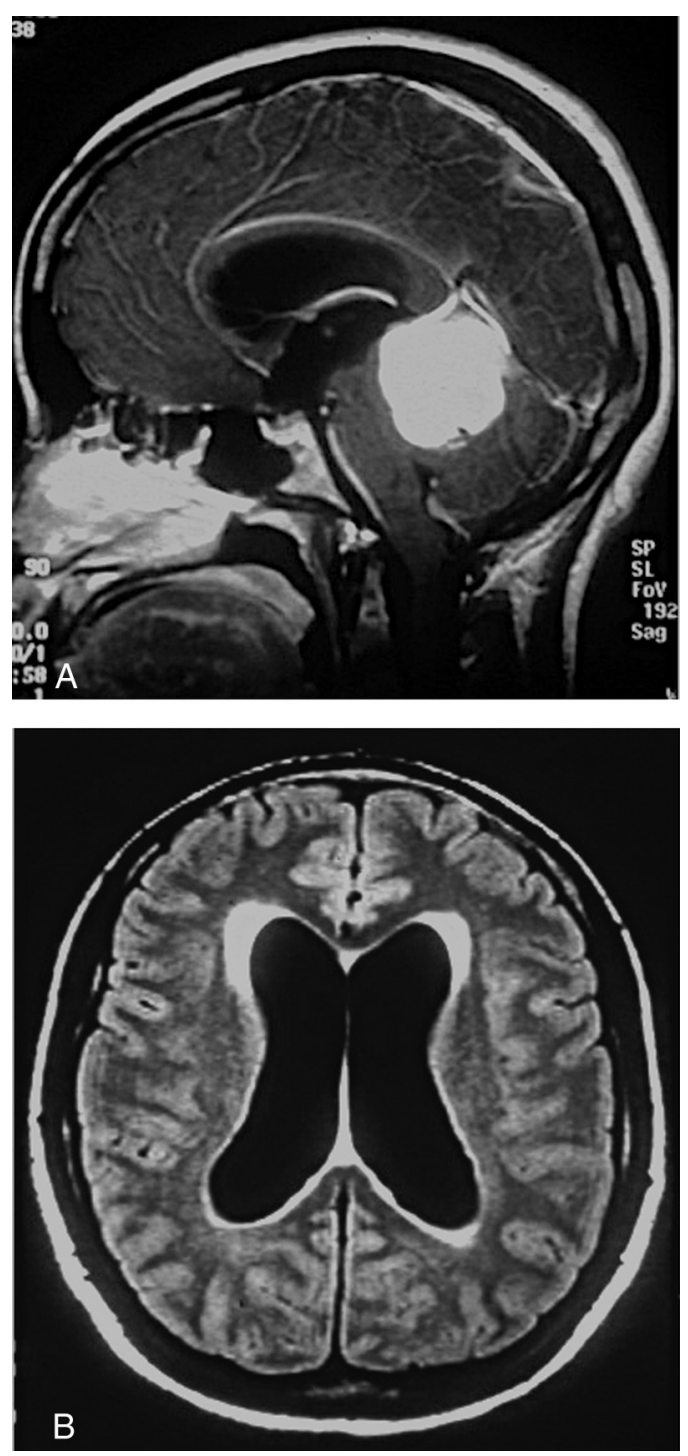

FIG 3. Interstitial edema shown on an axial FLAIR image from an obstructing juvenile pilocytic astrocytoma.

gestive of NPH, the first diagnostic test is usually MR imaging looking for ventricular dilation out of proportion to any sulcal enlargement (ie, the pattern of communicating hydrocephalus rather than atrophy). The amount of interstitial edema surrounding the lateral ventricles should be minimal to absent (which goes along with the normal mean intraventricular pressure). There is frequently associated evidence of deep white matter ischemia also known as small-vessel ischemia or leukoaraiosis (Fig 1A). ${ }^{11}$ The third ventricle walls, which generally bow inward (producing a waist), become parallel or even bowed out and may have a prominent CSF flow void that extends down through the aqueduct to the obex of the fourth ventricle (Fig 1).

The CSF flow void is indicative of hyperdynamic CSF flow similar to the flow voids seen in arteries on MR imaging. The extent of the CSF flow void on conventional spin-echo images in the past was found to correlate with a successful response to ventriculoperitoneal shunting. ${ }^{12}$ Unfortunately, the more modern MR imaging techniques such as fast/turbo spin-echo are much more intrinsically flow-compensated and do not have the same

AJNR Am J Neuroradiol 36:831-38 May 2015 www.ajnr.org 


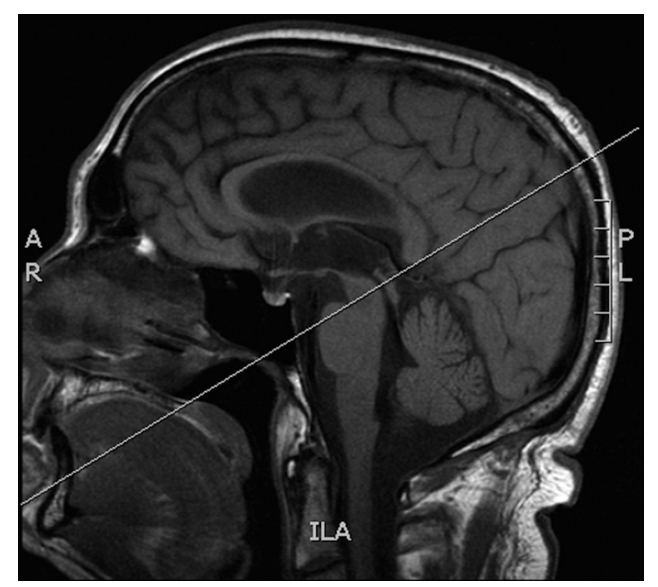

FIG 4. Slice positioning perpendicular to the midaqueduct for a phase-contrast CSF flow study.
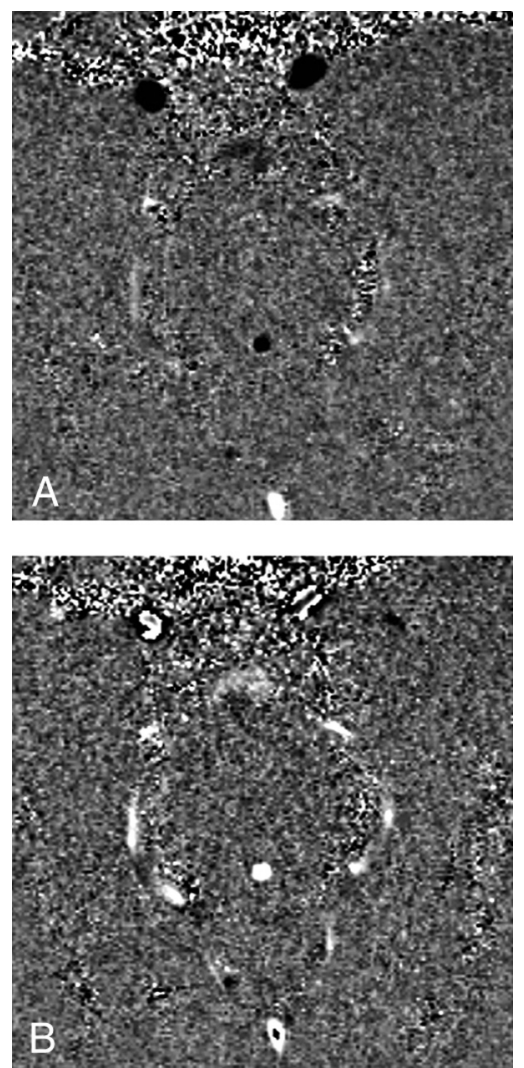

FIG 5. Phase-contrast images showing aqueductal flow up during diastole (black) and down during systole (white).

flow void as seen in the early days of MR imaging. These features led to the development of more sophisticated phase-contrast (PC) MR imaging techniques to evaluate CSF flow for the selection of appropriately symptomatic patients for possible ventriculoperitoneal shunting for $\mathrm{NPH}^{13}$

With PC-MR imaging, the slice is positioned in an angled axial plane so that it is perpendicular to the aqueduct (Fig 4). Higher resolution is better because the aqueduct is such a small structure. We use a $512 \times 512$ matrix over a $16-\mathrm{cm}$ FOV, achieving a spatial resolution of $312 \mu \mathrm{m}(0.312 \mathrm{~mm})$ (Fig 5$)$, though some use lower spatial resolution to save time. Like phase-contrast MR angiogra-

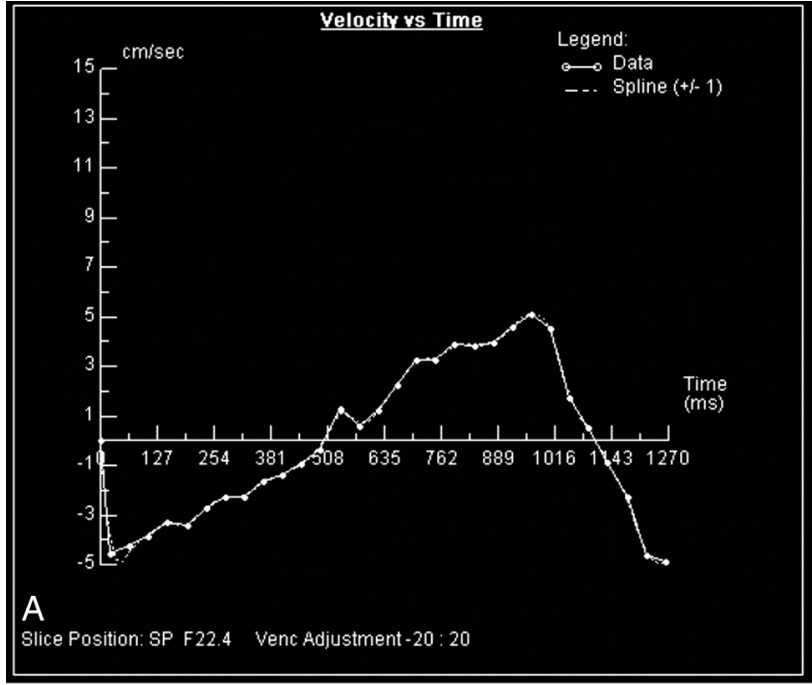

\begin{tabular}{|c|c|c|}
\hline Slice Position: SP F22.4 & \multirow{2}{*}{\multicolumn{2}{|c|}{$\begin{array}{l}\text { Region: } 1 \\
\text { Venc Adjustment }-20 \mathrm{~cm} / \mathrm{sec} \quad 20 \mathrm{~cm} / \mathrm{sec}\end{array}$}} \\
\hline Range,ms: 0 to 1263 & & \\
\hline Body Surface Area (BSA): & -..- & \\
\hline \multicolumn{3}{|l|}{ Velocity } \\
\hline Peak Velocity: & 15.14 & $\mathrm{~cm} / \mathrm{sec}$ \\
\hline Average velocity: & -0.004 & cmisec \\
\hline \multicolumn{3}{|l|}{ Flow } \\
\hline Average Flow Over Range: & -0.001 & $\mathrm{~m} / \mathrm{sec}$ \\
\hline Average Flow Per Minute: & -..- & $1 \mathrm{~min}$ \\
\hline Forward Volume: & 0.255 & $\mathrm{ml}$ \\
\hline Reverse Volume: & 0.255 & $\mathrm{ml}$ \\
\hline Net Forward Volume: & -0.001 & $\mathrm{ml}$ \\
\hline Net Forward Volume i BSA: & $\cdots$ & $\mathrm{ml} / \mathrm{m}^{\wedge} 2$ \\
\hline \multicolumn{3}{|l|}{ Area } \\
\hline Average Area: & 0.150 & $\mathrm{~cm}^{n} 2$ \\
\hline Mininum Area: & 0.150 & $\mathrm{~cm}^{n 2}$ \\
\hline Maximum Area: & 0.150 & $\mathrm{~cm}^{n} 2$ \\
\hline S & & \\
\hline
\end{tabular}

FIG 6. Volumetric, almost sinusoidal, CSF flow through the aqueduct during 1 cardiac cycle. Integrating the areas under and over the horizontal zero flow line yields the volumes of CSF going caudad in systole and cephalad in diastole, respectively, as shown in the chart. These should be within $5 \%$, and their average is the CSF stroke volume.

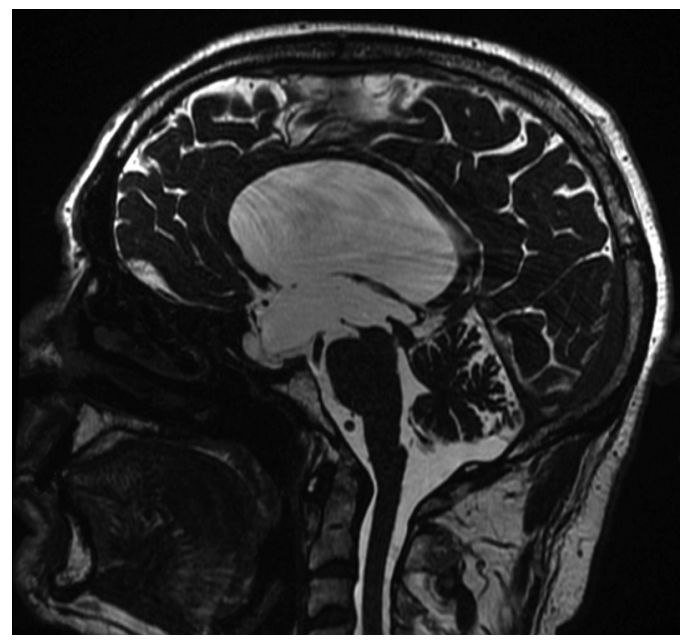

FIG 7. Midsagittal FIESTA image showing a web in the distal aqueduct. 
phy, the encoding velocity needs to be specified before the study being performed or aliasing will occur. Because most of these studies are performed by the MR imaging technologists without physician supervision, we use encoding velocities of 10,20 , and 30 $\mathrm{cm} / \mathrm{s}$ to balance aliasing versus sensitivity. We also use retrospective cardiac gating with either chest (electrocardiogram) leads or finger plethysmography. Most MR imaging systems today have automated software that calculates the volume of CSF flowing craniocaudad during systole and caudocraniad during diastole. Because the flow down and the flow up are within 5\% of each other (with a small net forward motion), we take the average and call it the "aqueductal CSF stroke volume" (ACSV). While our early phase-contrast CSF flow studies took 14 minutes to perform, ${ }^{14}$ modern techniques that have multiple views per TR (eg,

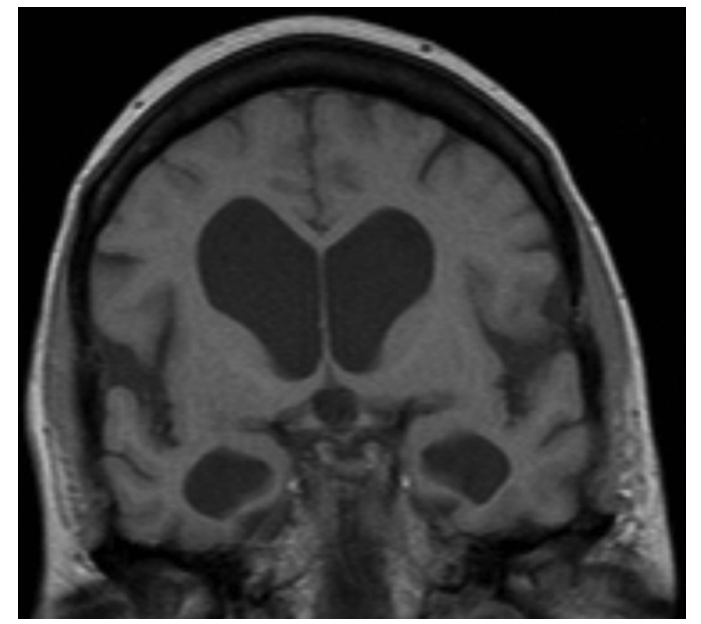

FIG 8. DESH pattern of NPH with ventriculomegaly, prominent Sylvian cisterns, and tight superior convexities.
NOVA; VasSol, River Forest, Illinois) take 1-2 minutes for each encoding velocity.

We have found that patients who respond to shunting for $\mathrm{NPH}$ have at least twice the ACSV of healthy elderly patients. This is because they have enlarged ventricles and minimal, if any, atrophy. As noted above, when the brain expands during systole in healthy elderly patients, it expands outward toward the SAS and inward toward the ventricles. In patients with early NPH, the brain is already expanded out against the inner table of the calvarium, so all systolic expansion is directed inward against the enlarged ventricles. This larger drumhead of the enlarged ventricles leads to hyperdynamic CSF flow through the aqueduct, which we measure as an elevated ACSV. If these patients are not shunted, they will eventually develop atrophy and the amount of systolic expansion and the ACSV will be reduced. Thus, hyperdynamic CSF flow indicates that atrophy has not yet taken place.

While different investigators have used different values for the ACSV appropriate for shunting, it is highly machine- and technique-dependent. Therefore, it is recommended that anyone wishing to use PC-MR imaging to diagnose shunt-responsive NPH first perform CSF flow studies on a number of healthy elderly patients without dilated ventricles to determine what is normal on that scanner. Then when a patient with suspected NPH is evaluated, an ACSV at least twice that value should be sought before recommending shunting.

While this last sentence suggests that a single number might be used to recommend shunting, this is obviously never the case in medicine. First the quality of the PC CSF flow technique must be evaluated. Lower encoding velocities tend to be more accurate but are more susceptible to velocity aliasing, while higher encoding velocities are less sensitive to aliasing but are noisier. The sinusoi-
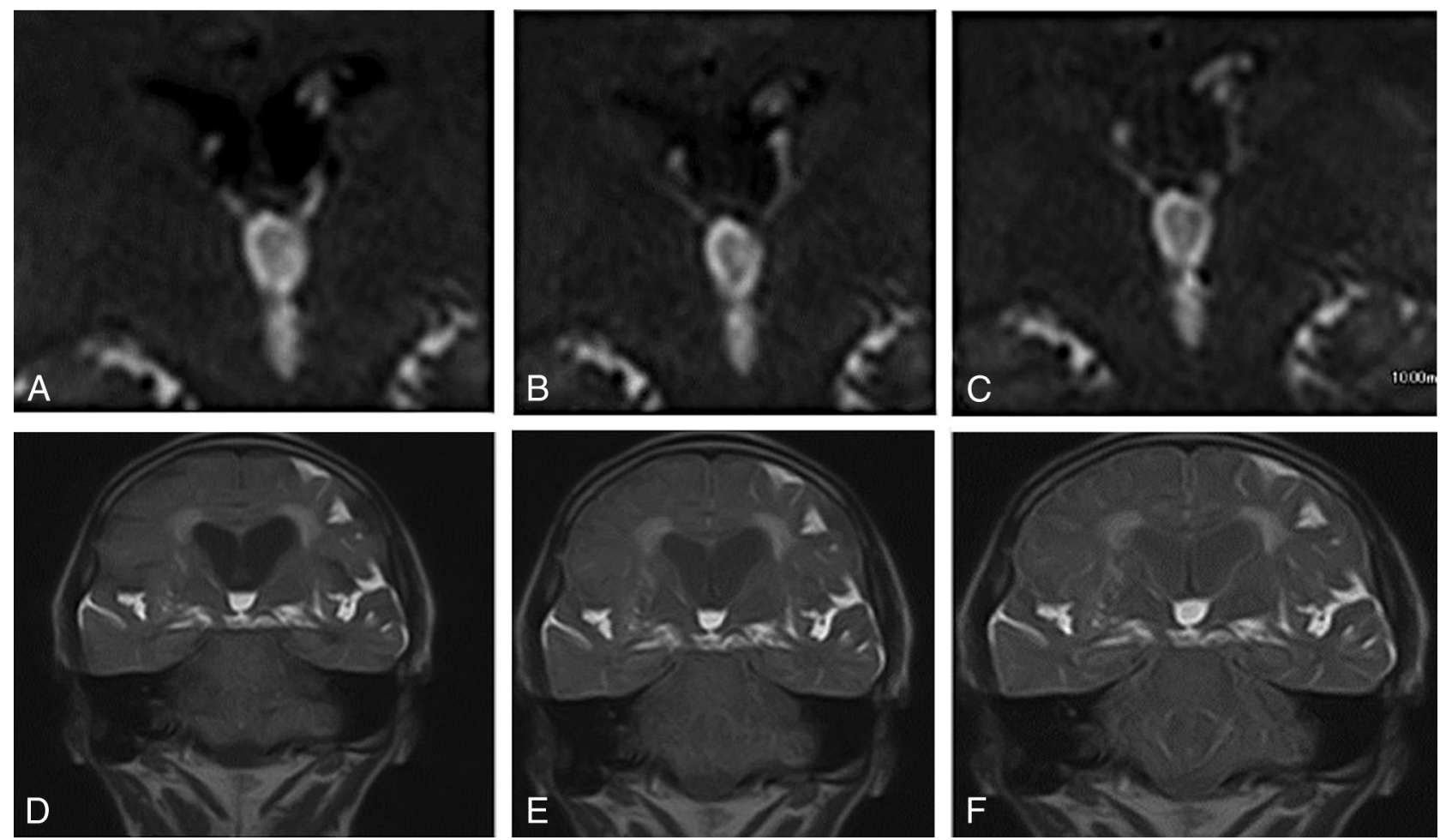

FIG 9. Time-SLIP in a healthy individual $(A-C)$ with lateral ventricular reflux and in a patient with NPH (D-F) without reflux. Images courtesy of Shinya Yamada, MD. 

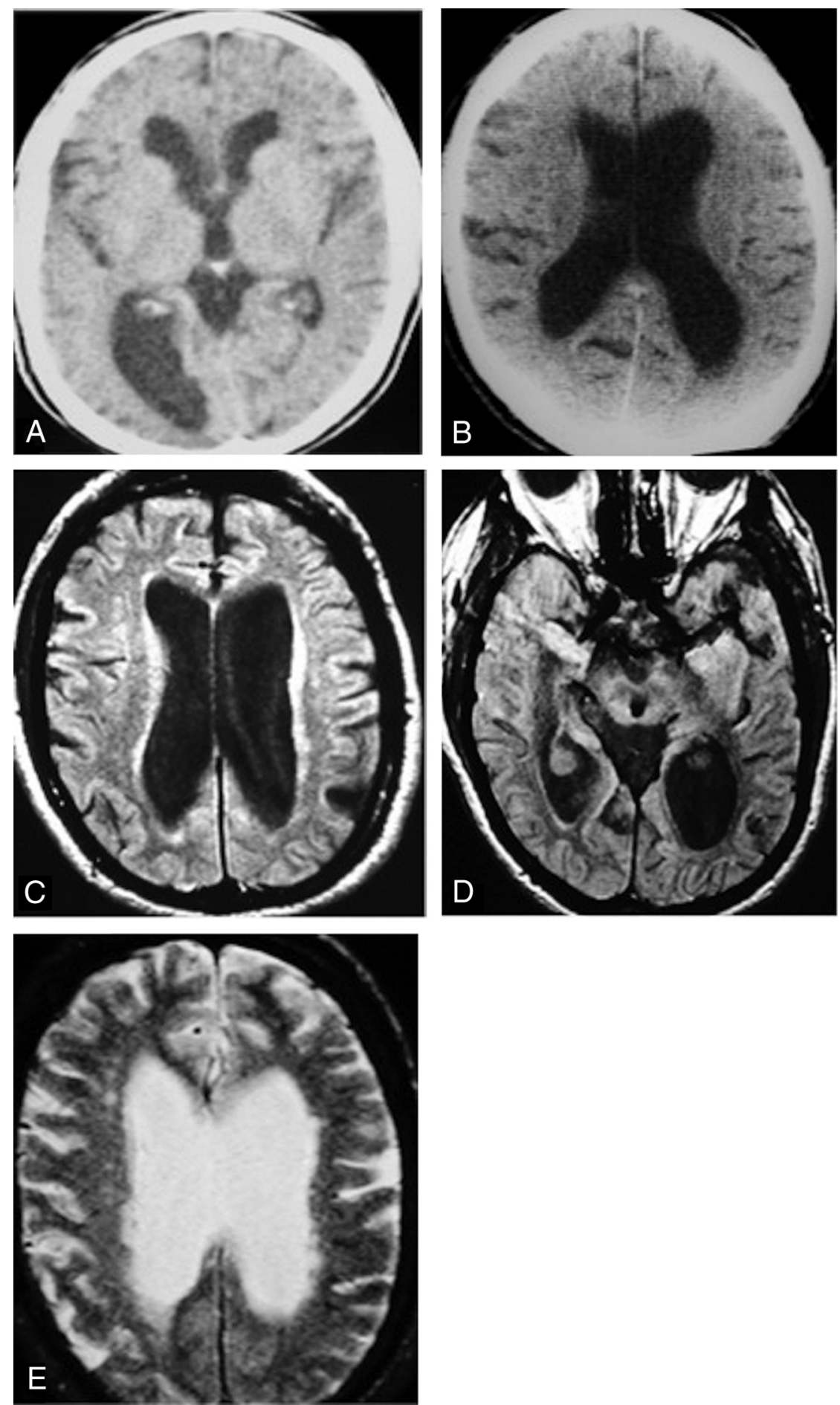

FIG 10. Future patient with NPH with 19 years of earlier imaging showing ventriculomegaly before symptoms of NPH. A, CT scan from 19 years earlier obtained for suspected giant cell arteritis shows mild ventriculomegaly. He was 67 years of age at this point and was walking 20 miles per day. $B$, At age 70 , he clearly has ventriculomegaly but no symptoms of NPH. $C-E$, Now at 76 years of age, MR imaging shows ventriculomegaly, DWMI, and an aqueductal CSF flow void. He will not develop symptoms of NPH for another 10 years. Reprinted with permission from Bradley et al. ${ }^{20}$ Copyright 2006 Wiley-Liss, Inc.

$\mathrm{NPH}$, it is most likely that the patient has already developed atrophy and will be less likely (but not impossible) to improve with a shunt. It has been shown that patients very early in their disease may not have developed hyperdynamic CSF flow yet and may benefit from a repeat study in 6 months. ${ }^{15}$

When we perform MR imaging for possible $\mathrm{NPH}$, we perform routine $\mathrm{MR}$ imaging of the brain, a PC-MR imaging CSF flow study, and bright CSF, thinsection sagittal imaging to evaluate possible aqueductal stenosis. Depending on the MR imaging vendor, this could be FIESTA (GE Healthcare, Milwaukee, Wisconsin) (Fig 7), TrueFISP (Siemens, Erlangen, Germany), or balanced fastfield echo (Philips Healthcare, Best, the Netherlands), but it is important to get a section thickness of $<1 \mathrm{~mm}$. Because aqueductal stenosis presents with the same clinical triad as NPH, plus chronic headaches, these patients are often referred for imaging to exclude NPH. As part of the routine MR imaging, we also perform a midcoronal sequence (either T1- or T2weighted) looking for the disproportionately enlarged subarachnoid space hydrocephalus (DESH) pattern, ${ }^{16}$ which is qualitatively large Sylvian cisterns and a tight superior convexity SAS (Fig 8), though we have not found this sign to be as useful as the ACSV in predicting shuntresponsive NPH.

In the United States, PC-MR imaging is often combined with a high-volume CSF tap test or the more invasive external lumbar drainage to evaluate a suitably symptomatic patient with NPH for ventriculoperitoneal shunting. In Europe, saline infusion is commonly used. In Japan, it has been reported that the tap test does not add any diagnostic value if there is a DESH pattern. ${ }^{17}$

While most experience demonstrating hyperdynamic CSF flow in NPH was with PC-MR imaging, there is a new technique called time-spatial labeling inversion pulse (Time-SLIP), which clearly has some utility. ${ }^{18}$ Time-SLIP is a CSF tagging technique similar to arterial spin-labeldal volumetric CSF flow curve (Fig 6) should be evaluated for aliasing and to be certain that the area under the curve above the zero flow line (diastole) equals the area below the line (systole). If systolic flow is greater than diastolic flow, it is possible that the retrospective cardiac gating is not adequately sampling diastole.

If the ACSV is not twice normal in a patient with symptomatic ing, which can show increased CSF displacement through the aqueduct in NPH or no displacement in aqueductal stenosis. When it is applied in the coronal plane through the foramen of Monro, CSF regurgitation into the lateral ventricles can be seen in healthy patients during diastole (Fig 9, $A-C$ ); however, there is very little regurgitant flow in NPH (Fig 9, $D-F)$. 


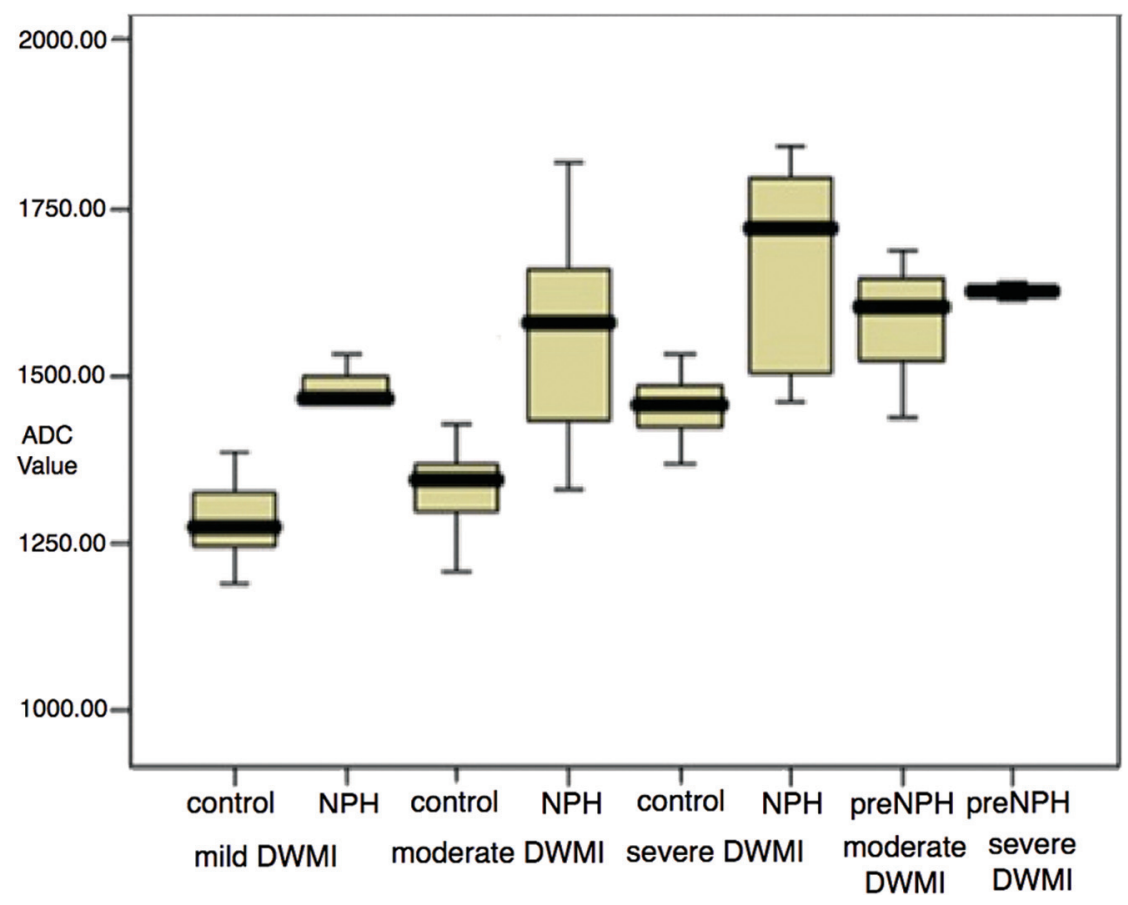

FIG 11. Apparent diffusion coefficient versus the degree of DWMI in NPH and age-matched controls, showing significantly higher ADC (indicating higher water content) in patients with NPH versus controls for a given degree of DWMI. Reprinted with permission from Bradley et al. ${ }^{20}$ Copyright 2006 Wiley-Liss, Inc.

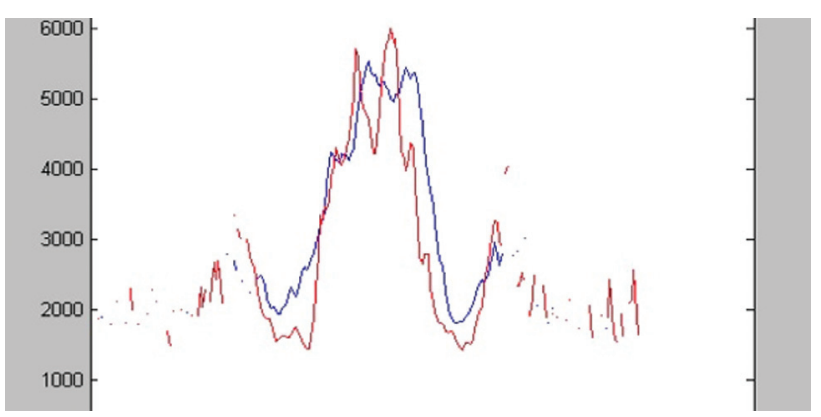

FIG 12. ADC profile in the mid-coronal plane in healthy individuals (red) and patients with NPH (blue). The central double peak is the lateral ventricles. Note the higher water content in the extracellular space next to the ventricles in the NPH group, possibly due to impaired centrifugal flow from DWMI. Reprinted with permission from Bradley et al. ${ }^{20}$ Copyright 2006 Wiley-Liss, Inc.

\section{Possible Etiology of Idiopathic NPH}

Because I have used various MR imaging indicators of hyperdynamic CSF flow for $>30$ years now, it is tempting to speculate on the etiology of "idiopathic" NPH. We know that patients with shunt-responsive NPH have both hyperdynamic CSF flow and deep white matter ischemia (DWMI) (Fig 1). We also know that patients with NPH have significantly $(P<.003)$ larger intracranial volumes than age- and sex-matched controls and have had dilated ventricles for years before becoming symptomatic (Fig 10). ${ }^{19}$ The increased intracranial volume raises the possibility that perhaps these patients had benign external hydrocephalus as infants. $^{20}$

With decreased uptake of CSF by the arachnoidal granulations after passing through the usual pathway (ie, the fourth ventricle outlet foramina), such patients might have developed a parallel pathway for CSF resorption, similar to a parallel electrical circuit with a fixed voltage drop that can now conduct twice as much current. A potential parallel pathway for CSF resorption would be the extracellular space of the brain. Thus CSF would cross the ependyma into the ISP and be transported out the venous Virchow-Robin spaces via the aquaporin-4 water channels. These patients would continue with this dual pattern of CSF resorption, with some of the CSF gliding over the myelin lipid, until their elderly years when they develop DWMI, which might be considered the second "hit" in this disease. ${ }^{20}$

The histopathologic hallmark of DWMI is myelin pallor. ${ }^{21}$ With less lipid, there is more water and high signal on T2weighted and FLAIR images (Fig 1). The outward-flowing CSF in the ISP of the brain is no longer gliding over the myelin lipid but is now attracted to the myelin protein. This attraction between the polar water molecules of the CSF and the charged side groups of the myelin protein increases the resistance to CSF outflow via the extracellular or interstitial space of the brain. The outflowing CSF is essentially dammed up by the DWMI and backs up; this process leads to hydrocephalus. According to Hakim's hypothesis, ${ }^{8,9}$ the tangential shearing forces near the ventricles lead to gait disturbance and the subsequent radial shearing forces compress the cortex against the inner table of the calvarium leads to dementia.

In support of the above hypothesis is the finding that there is more water in the extracellular space of the brain in patients with $\mathrm{NPH}$ than in age-matched controls. ${ }^{20}$ This finding is because the apparent diffusion coefficient from diffusion-weighted imaging is elevated compared with that in healthy individuals. While DWMI has a higher water content than normal brain as noted above, the ADC is statistically higher for a given degree of DWMI in patients with NPH than in healthy elderly patients (Fig 11). It is also highest in the periventricular region, supporting the concept that the DWMI is damming up the outflowing CSF (Fig 12).

Note that the right-hand columns in Fig 11 are labeled "preNPH.” These patients were considered healthy elderly controls without symptoms of NPH. They may fall into the same category as the patient illustrated in Fig 10, who had enlarged ventricles 19 years before he developed symptoms of NPH. The Japanese call this AVIM (asymptomatic ventriculomegaly with features of idiopathic NPH on MR imaging). It is important to observe these patients for the potential development of a future gait disturbance.

\section{ACKNOWLEDGMENTS}

I thank Abdulrahman A. Almutairi, MD, for assistance with the images. 


\section{REFERENCES}

1. Iliff JJ, Wang M, Liao Y, et al. A paravascular pathway facilitates CSF flow through the brain parenchyma and the clearance of interstitial solutes, including amyloid $\boldsymbol{\beta}$. Sci Transl Med 2012;4:147ra111

2. Iliff JJ, Lee H, Yu M. Brain-wide pathway for waste clearance captured by contrast-enhanced MRI. J Clin Invest 2013;123:1299-309

3. Iliff JJ, Wang M, Zeppenfeld DM, et al. Cerebral arterial pulsation drives paravascular CSF-interstitial fluid exchange in the murine brain. J Neurosci 2013;33:18190-99

4. Bradley WG Jr, Kortman KE, Burgoyne B. Flowing cerebrospinal fluid in normal and hydrocephalic states: appearance on MR images. Radiology 1986;159:611-16

5. Mokri B. The Monro-Kellie hypothesis: applications in CSF volume depletion. Neurology 2001;56:1746-48

6. Zahl SM, Egge A, Helseth E, et al. Benign external hydrocephalus: a review, with emphasis on management. Neurosurg Rev 2011;34: 417-32

7. Nickel RE, Gallenstein JS. Developmental prognosis for infants with benign enlargement of the subarachnoid spaces. Dev Med Child Neurol 1987;29:181-86

8. Hakim S, Adams RD. The special clinical problem of symptomatic hydrocephalus with normal cerebrospinal fluid: observations on cerebrospinal fluid hydrodynamics. J Neurol Sci 1965;2:307-27

9. Adams RD, Fisher CM, Hakim S, et al. Symptomatic occult hydrocephalus with "normal" cerebrospinal fluid pressure: a treatable syndrome. N Engl J Med 1965;273:117-26

10. Spetzler RF. Normal pressure hydrocephalus. Barrow Quarterly 2003;19:1

11. Bradley WG Jr, Whittemore AR, Watanabe AS, et al. Association of deep white matter infarction with chronic communicating hydrocephalus: implications regarding the possible origin of normal pressure hydrocephalus. AJNR Am J Neuroradiol 1991;12:31-39

12. Bradley WG Jr, Whittemore AR, Kortman KE, et al. Marked CSF flow void: an indicator of successful shunting in patients with suspected normal pressure hydrocephalus. Radiology 1991;178:459-66

13. Nitz WR, Bradley WG Jr, Watanabe AS, et al. Flow dynamics of cerebrospinal fluid: assessment with phase-contrast velocity MR imaging performed with retrospective cardiac gating. Radiology 1992; 183:395-405

14. Bradley WG Jr, Scalzo D, Queralt J, et al. Normal-pressure hydrocephalus: evaluation with cerebrospinal fluid flow measurements at MR imaging. Radiology 1996;198:523-29

15. Scollato A, Tenenbaum R, Bahl G, et al. Changes in aqueductal CSF stroke volume and progression of symptoms in patients with unshunted idiopathic normal pressure hydrocephalus. AJNR Am J Neuroradiol 2008;29:192-97

16. Hashimoto M, Ishikawa M, Mori E, et al. Study of INPH on neurological improvement (SINPHONI): diagnosis of idiopathic normal pressure hydrocephalus is supported by MRI-based scheme: a prospective cohort study. Cerebrospinal Fluid Res 2010;7:18

17. Ishikawa M, Hashimoto M, Mori E, et al. The value of the cerebrospinal fluid tap test for predicting shunt effectiveness in idiopathic normal pressure hydrocephalus. Fluids Barriers CNS 2012;9:1

18. Yamada S, Miyazaki M, Kanazawa H, et al. Visualization of cerebrospinal fluid movement with spin labeling at MR imaging: preliminary results in normal and pathophysiologic conditions. Radiology 2008;249:644-52

19. Bradley WG, Safar FG, Furtado C, et al. Increased intracranial volume in normal pressure hydrocephalus: a clue to the etiology of “idiopathic" NPH? AJNR Am J Neuroradiol 2004;25:1479-84

20. Bradley WG Jr, Bahl G, Alksne JE, et al. Idiopathic normal pressure hydrocephalus may be a "two hit" disease: benign external hydrocephalus in infancy followed by deep white matter ischemia in late adulthood. J Magn Reson Imaging 2006;24:747-55

21. Marshall VG, Bradley WG Jr, Marshall CE, et al. Deep white matter infarction: correlation of MR imaging and histopathologic findings. Radiology 1988;167:517-22 